\title{
Image recognition algorithm based on Parameter Optimization of gravitational search

\author{
LEI Hu $\mathrm{Hu}^{1, \mathrm{a}}$ \\ ${ }^{1}$ Xi'an Fanyi University, Xi'an, Shaanxi, 710105, China \\ a email:505410068@qq.com
}

Keywords: Gravitational search algorithm; Parameter optimization; Image recognition

\begin{abstract}
Image characteristic value extracted by gravitational search algorithm improves the image retrieval accuracy. The distance between the image characteristic values is as the input value, accuracy of image recognition is calculated by pulse coupled network variates optimized of Gravitational search algorithm. The retrieval accuracy of pulse coupled network optimized by gravitational search algorithm improves by $7 \%$ at least than method of relevance feedback, color edge combined discrete wavelet transform.
\end{abstract}

\section{Introduction}

With the rapid development of the Internet, digital image real-time processing and retrieval becomes the most important medium of communication in daily life, Compared with the availability and the rapid development of hardware, software usability of management atlas is limited, image retrieval(IRBC) based on content is a common method to retrieve a specific image, and IRBC is one of the challenging research topic in image retrieval and pattern recognition and data mining area.

IRBC system has existed for more than thirty years, the first commercial IRBC system such as QBIC of IBM. There are many academic research based on color features, such as RGB, HSV, and texture image content on such as Candid, Photobook, etc. Different IRBC system is different, but the application framework includes two phases, namely characteristic expression and similarity measure. Therefore, generally focuses on IRBC method can be divided into the characteristics of expression and the subsequent retrieval method.

IRBC method retrieves image by the visual features of the image, its efficiency mainly depends on the image characteristics of forms, such as based on color feature and texture to describe the Candid, Photobook system of image content. Agarwal S et al. proposed the image retrieval technology based on image texture and shape, but it can't accurately describe the color changes based on edge histogram feature vector. Xiang-li xu et al. proposed IRBC method based on particle swarm optimization, it combines with particle swarm optimization and user feedback process and avoids the influence to user cognition in initial retrieval, however the method sometimes appears with the problem of insufficient sample. Li G proposed an improved correlation feedback method of IRBC by using the image of the observation error function to choose the corresponding regression function, this method can take care of image semantic features and image space geometry, but it ignores semantic gap problem between visual features and semantic category. Mr. Choudhary R et al. represent Color features by using Color Metric) and represent the texture by using Local Binary patterns (LBP), the method is very pay attention to the representation of object retrieval image, but it ignores the subsequent retrieval efficiency. In order to solve color distortion of lossy compression, Mr. Choudhary R et al. proposed the image color information by using the Gaussian Mixture Model (GMM) and assess the color uniformity, and solve color distortion of lossy compression, however, it is slowly for nonlinear problem converged because of the initial value of the GMM Model is set difficulty.

In this paper, pulse coupled network $(\mathrm{PCN})$ parameters are optimized by the gravitational search algorithm(GSA), characteristic generated by the PCN is used to visual feature of vector image, in 
order to improve retrieval efficiency of the definition categories of matching after classifying visual characteristics.

\section{Parameters optimized algorithm of PCN}

Fig.1 shows that PCN includes two components:links and feedback, the value of the neuron will influence the value of the next neuron by using threshold and step function as working of PCN in iterative way.

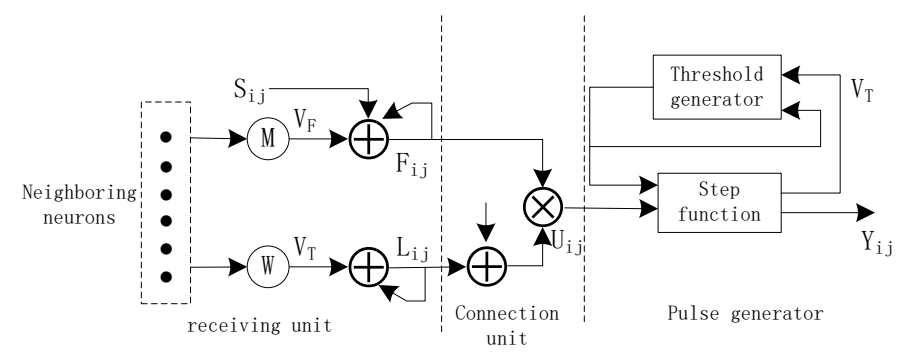

Fig. 1 PCN model

$\mathrm{L}_{\mathrm{ij}}$ is a link value of neurons, $\mathrm{Y}_{\mathrm{ij}}$ is output value, $\mathrm{VF}, \mathrm{AF}, \mathrm{VT}, \mathrm{AT}, \mathrm{VL}, \mathrm{AL}$ and $\beta$ are constant, $\mathrm{n}$ is the number of iterations, and $F_{i j}$ is feedback value, $A_{i j}$ is the strength of the digital image pixel, $U_{i j}$ is a state of neurons, Eq. 1 is the relationship between output value and links, Eq. 2 is the relation of back part of the receiving neuron feedback value and output value, Eq.3 is the relationship of the output value in links and feedback part, such as, the threshold value $T_{i j}$ can be expressed as Eq.4.

$$
\begin{aligned}
& L_{i j}(n)=L_{i j}(n-1) * e^{\frac{-1}{A L}}+V L * Y_{i j}(n-1) \\
& F_{i j}(n)=F_{i j}(n-1) * e^{\frac{-1}{A F}}+V F * Y_{i j}(n-1)+A_{i j} \\
& U_{i j}(n)=F_{i j}(n)+\left(1+\beta * L_{i j}(n)\right) \\
& T_{i j}(n)=T_{i j}(n-1) * e^{\frac{-1}{A T}}+V T * Y_{i j}(n)
\end{aligned}
$$

This shows that parameter values of AL and AF and AT and VL and VF and VL and VT and $\beta$ have a great influence on the PCN generated characteristic value. Therefore, choose the optimal parameter values to ensure the quality of characteristic value.

\section{Parameters optimized by the gravitational search algorithm}

Gravitational attraction between the particles in gravitational search algorithm, which made population move at the direction of the particle with large quality, the key problem of the algorithm is to determine particle position.

Attraction and inertial mass can be calculated by adaptive function, and the greater attraction of the particle moves slower speed than before, it assumes that attraction and inertial mass in GSA algorithm is equal, and $M_{p i}=M_{a j}=M_{i}, i=1,2, \ldots, n \cdot M_{i}(t)$ is quality and $f_{i}(t)$ is fitness function in the $\mathrm{i}$ times particle at the $\mathrm{t}$ times iteration, $W(t)$ and $B(t)$ are the worst fitness function and the best fitness function of Particle in the $t$ times iteration, $M_{i}(t)$ is the quality of the $i$ times particle defined as Eq.5.

$$
M_{i}(t)=\frac{f_{i}(t)-w(t)}{(B(t)-W(t)) \times \sum_{j=1}^{n} q_{j}(t)}
$$

$M_{p i}(t)$ is the passive gravitational mass of the ith particle, $M_{a j}(t)$ is active gravitational mass of the jth particle, $G(t)$ and $\wp$ are constant, Rij $(\mathrm{t})$ is the Euclidean distance of particle $\mathrm{i}$ and particle $\mathrm{j}$. $F_{i j}{ }^{d}(t)$ is gravity of particle $\mathrm{i}$, which is defined as Eq.6. 


$$
F_{i j}{ }^{d}(t)=G(t) \frac{M_{p i}(t) \times M_{a j}(t)}{R_{i j}(t)+\wp}\left(x_{j}{ }^{d}(t)-x_{i}{ }^{d}(t)\right)
$$

Value of rand is the range as a random number between $[0,1]$, which express gravity of particle $\mathrm{i}$ in the Dth dimension. The value of rand is to make GSA reach the characteristics of random:

$$
F_{i}^{d}(t)=\sum_{j \in k b, j \neq i} \text { rand } \bullet F_{i j}{ }^{d}(t)
$$

If $R_{i j}(t)=\left\|X_{i}(t), X_{j}(t)\right\|$, the acceleration of the i particle on $\mathrm{d}$ dimension is defined as Eq.7

$$
a_{i}^{d}(t)=\frac{F_{i}^{d}(t)}{M_{i}(t)}=\sum_{j \neq i} \text { rand } \times G(t) \times \frac{M_{j}(t)}{R_{i j}+\varepsilon} \times\left(x_{j}^{d}(t)-x_{i}^{d}(t)\right)
$$

The speed of the particle $\mathrm{i}$ in the $\mathrm{t}+1$ time on $\mathrm{d}$ dimension is defined as Eq.8, the position of the particle $\mathrm{i}$ in $\mathrm{t}+1$ time on dimension is defined as Eq.9.

$$
\begin{aligned}
v_{i}^{d}(t+1) & =\text { rand } \times v_{i}^{d}(t)+a_{i}^{d}(t) \\
x_{i}^{d}(t) & =x_{i}^{d}(t-1)+v_{i}^{d}(t)
\end{aligned}
$$

The quality of characters generated in PCN is improved by parameter optimization mechanism. It assumes that AL and AT and VL and VT and $\beta$ are optimized parameters. $X$ is characteristic of the original image, and $\mathrm{Y}$ is the characteristic of the target image, $\mathrm{k}$ is the length of characteristic, adaptive function is calculated by Euclidean distance as Eq.10.

$$
\operatorname{eucl}(x, y)=\sqrt{\sum_{i=1}^{k}\left(X_{i}-Y_{i}\right)^{2}}
$$

The retrieve process of classified digital image in PCN after optimized pattern in GSA is shown in fig.2. the process realizes parameter optimization by predefined number of iterations, which determine the optimal characteristic value for all images. the each characters of digital image generated by PCN optimization processing is saved, and this process will determine the minimum distance between digital image by comparing the value of characters between similar digital images and put in storage.

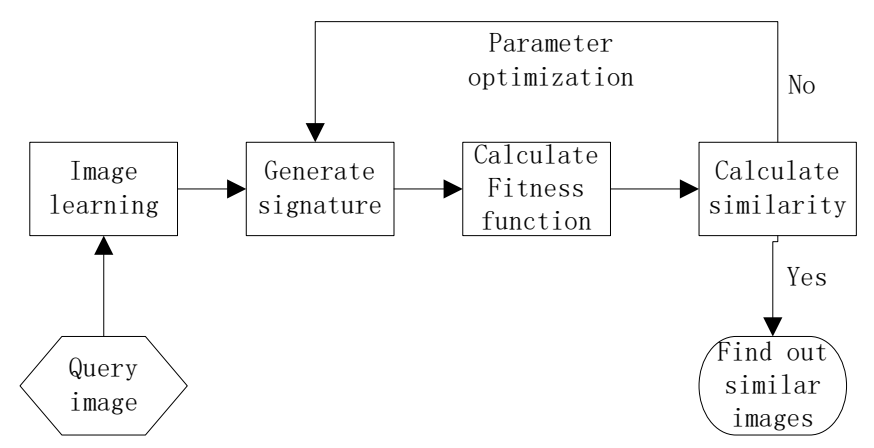

Fig. 2 Image retrieval process

\section{Simulations and results}

In Windows 7 system and matlab2010 environment, The experimental simulation uses the Corel image database and choose 10 types of semantic images including the Africans, elephants, horses, flowers, food, mountains, buildings and buses etc al.. Image size is transformed into size of $256 \times$ 256 pixels with JPEG format, the PCN training process uses eight pictures each class randomly selected from Corel images. Through the experimental simulation environment, using matlab2010 programming, it is to find out similar images by comparing the distance of the image and the image in standard database. 
Recall and precision are the evaluation criteria of image retrieval based-content, Assuming a_num is image number of the accurate retrieval, $r$ num is for the total of images retrieved, all_um is the total of retrieved image in the database, $\mathrm{pr}$ is expressed $\frac{a_{-} \text {num }}{r_{-} \text {num }}$, re is expressed $\frac{r_{-} \text {num }}{a 11_{-} \text {num }}$.

Internal behavior of PCN depends defined value on the user, the method uses the characteristics with the length of 70 yards. By detecting activity of single neuron in the PCN, it is expressed the change process of $\mathrm{L}, \mathrm{F}$ and $\mathrm{T}$, it can be seen that the feedback values increase gradually until over the threshold. The continued growth of the threshold will prevent neurons trigger again. A moment later, the threshold is reduced, neurons signal pulse is allowed again. The output pulse signal makes PCN output series of binary image, each image represents a set of pixel sets and corresponds to the neurons inspired with 300 times iterations.

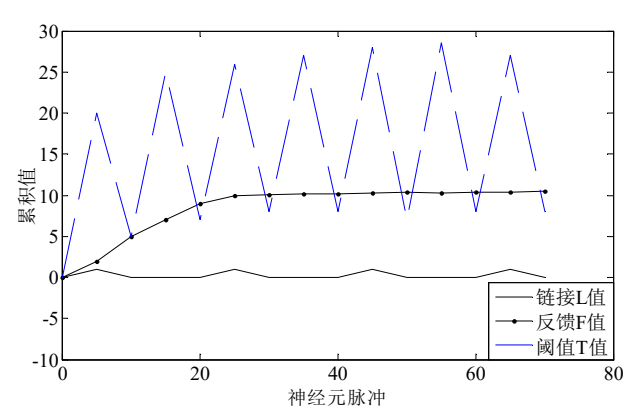

Fig. 3 cumulative value of single neuron

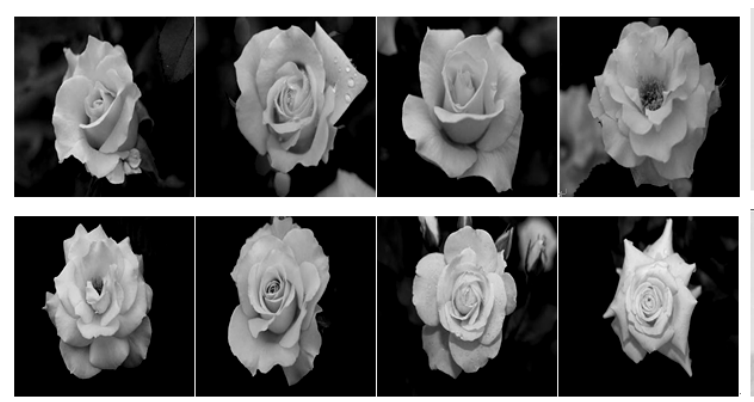

Fig. 5 Image sample sequence in Corel database

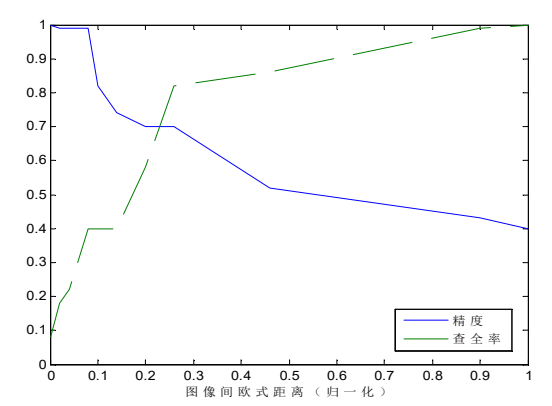

Fig. 4 The performance evaluation of the image retrieval (300 iterations)

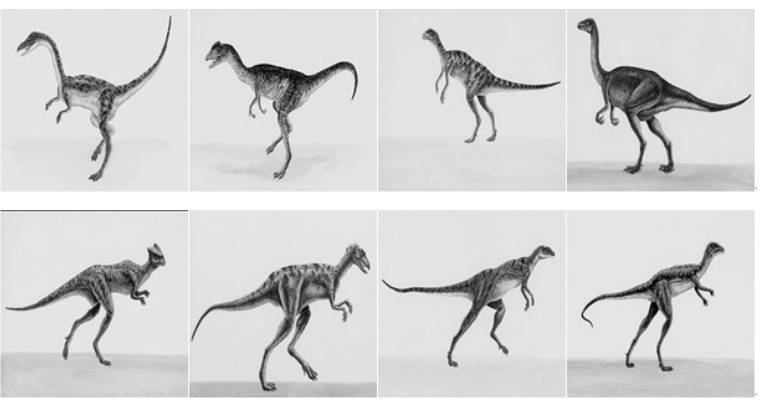

As shown in fig.4, the experimental result uses learned samples as input sequence. It can be seen that the recall rate increased and accuracy decreased with the increase of the distance between the input image and image matching. Relevant images retrieved When the distance between the input image and matching image reduces. A large amount of relevant images is retrieved by increasing the distance, which will lead to lower precision. On the other hand, the recall rate increases when uncorrelated images in image database increases. Therefore, the recall rate remained at a certain point at some point, because the most relevant images have retrieved at this point. The sample image sequence have been learned in fig.5.

It gives the comparison results of recall ratio and precision with the method of GSA,PCN, improve the irrelevant feedback method (IRF), and color edge combining discrete wavelet transform method (CE-DWT) in Fig.6 and Fig.7. The results show that the precision of GSA-PCN is better over $7 \%$ than precision of IRF and CE-DWT, it shows that the method of GSA-PCN has more advantages than others method, recall ratio is increased and the precision is reduced with an increase in the number of retrieving images.

\begin{tabular}{|c|c|c|c|c|c|c|}
\hline Algorithm & 30pics & 50pics & 70pics & 90pics & 110pics & 130pics \\
\hline IRF & $8.0 \%$ & $9.0 \%$ & $17.0 \%$ & $21.0 \%$ & $26.0 \%$ & $28.0 \%$ \\
\hline CE-I & 8.2 & & & $21.8 \%$ & $26.6 \%$ & $31.5 \%$ \\
\hline GSA-PCN & $8.6 \%$ & $15.4 \%$ & $22.4 \%$ & $29.4 \%$ & $35.4 \%$ & $38.7 \%$ \\
\hline
\end{tabular}

Fig. 6 The relationship between the average recall and retrieve images 


\begin{tabular}{|l|l|l|l|l|r|r|}
\hline algorithm & 30pics & 50pics & 70pics & 90pics & 110pics & 130pics \\
\hline IRF & $60.5 \%$ & $54.0 \%$ & $50.0 \%$ & $47.6 \%$ & $43.6 \%$ & $42.3 \%$ \\
\hline CE-DWT & $64.5 \%$ & $59.4 \%$ & $54.6 \%$ & $51.1 \%$ & $46.4 \%$ & $44.8 \%$ \\
\hline GSA-PCN & $82.1 \%$ & $74.8 \%$ & $71.1 \%$ & $67.8 \%$ & $64.8 \%$ & $60.2 \%$ \\
\hline
\end{tabular}

Fig. 7 The relationship between the average precision and retrieve images Comparison of three methods on Recall and precision

\section{Conclusions}

For image feature value extracted by PCN is not affected by rotation, scaling and tilt, it is to reduce storage space and processing costs of related images by classification, the growth in dimension of the search space increases the calculation time. The GSA-PCN method proposed in this paper uses attribute code fixed length and minimizes search the related image number is used to improve retrieval performance, which improves the efficiency of image recognition.

\section{Acknowledgement}

In this paper, the research was sponsored by the science research projects of education department in shaanxi province government(Project No. 16JK2078) and key project of education department in shaanxi provincial government (Project No.13BZ69).

\section{References}

[1] Jun-ding Sun. Image feature extraction and retrieval technology [D]. Electronic industry press, 2015.7.

[2] Ji-cang Lu,Fen-lin Liu,Xiang-yang Luo et al. Recognition of PQ stego images base on identifiable statistical feature[J].Journal on Communications, 2015,3(3) :2015068(1)- 2015068(10).

[3] Jiang-bo Yu,Hou-jin Chen, Wei Wang et al. Parameter determination of pulse coupled neural network in image processing[J]. Acta electronica sinica,2008,1(1):81-85

[4] Kodovsky J,Fridrich J.Rich models for steganalysis of digital images[J].IEEE Transactions on information Forensics and Security,2012,7(3):868-882.

[5] Shi-jiang Zhao,et al. A study of a new image segmentation algorithm based on PCNN[J]. Acta electronica Sinica,2005,33(7):1342-1344.

[6] He X F,Cai D,Han J. Learning a maximum margin sub-space for image retrieval.IEEE Transactons on Knowledge and Data Engineering,Feb.2008,20(2):189-201.

[7] Chen-hang Lin, Zhi-bin Pan, Bin Zou. Color image retrieval algorithm based on Global and local color features[J]. Microelectronics \& computer,2012.4(29):104-109.

[8]Yoon J, Kweon I. color image segmentation considering of human sensitivity for color pattern variations[J].SPIE,2001,4572:269-278. 